\title{
Noninvasive imaging of myocardial angiogenesis following experimental myocardial infarction
}

David F. Meoli, 1 Mehran M. Sadeghi, 1,2 Svetlana Krassilnikova, 1,2 Brian N. Bourke, Frank J. Giordano, ${ }^{1}$ Donald P. Dione, ${ }^{1}$ Haili Su, ${ }^{1}$ D. Scott Edwards, ${ }^{3}$ Shuang Liu, ${ }^{3}$ Thomas D. Harris, ${ }^{3}$ Joseph A. Madri, ${ }^{4}$ Barry L. Zaret, ${ }^{1,5}$ and Albert J. Sinusas ${ }^{1,5}$

${ }^{1}$ Division of Cardiovascular Medicine, Department of Internal Medicine, Yale University School of Medicine, New Haven, Connecticut, USA. ${ }^{2}$ Section of Cardiology, Department of Veterans Affairs Connecticut Healthcare Center, West Haven, Connecticut, USA. ${ }^{3 B}$ Bistol-Myers Squibb, North Billerica, Massachusetts, USA. ${ }^{4}$ Department of Pathology and ${ }^{5}$ Department of Diagnostic Radiology, Yale University School of Medicine, New Haven, Connecticut, USA

\begin{abstract}
Noninvasive imaging strategies will be critical for defining the temporal characteristics of angiogenesis and assessing efficacy of angiogenic therapies. The $\alpha v \beta 3$ integrin is expressed in angiogenic vessels and represents a potential novel target for imaging myocardial angiogenesis. We demonstrated the localization of an indium111-labeled (111In-labeled) $\alpha v \beta 3$-targeted agent in the region of injury-induced angiogenesis in a chronic rat model of infarction. The specificity of the targeted $\alpha v \beta 3$-imaging agent for angiogenesis was established using a nonspecific control agent. The potential of this radiolabeled $\alpha \mathrm{v} \beta 3$-targeted agent for in vivo imaging was then confirmed in a canine model of postinfarction angiogenesis. Serial in vivo dual-isotope single-photon emission-computed tomographic (SPECT) imaging with the ${ }^{111}$ In-labeled $\alpha v \beta 3$-targeted agent demonstrated focal radiotracer uptake in hypoperfused regions where angiogenesis was stimulated. There was a fourfold increase in myocardial radiotracer uptake in the infarct region associated with histological evidence of angiogenesis and increased expression of the $\alpha v \beta 3$ integrin. Thus, angiogenesis in the heart can be imaged noninvasively with an ${ }^{111}$ In-labeled $\alpha v \beta 3$-targeted agent. The noninvasive evaluation of angiogenesis may have important implications for risk stratification of patients following myocardial infarction. This approach may also have significant clinical utility for noninvasively tracking therapeutic myocardial angiogenesis.
\end{abstract}

\section{Introduction}

Angiogenesis represents the formation of new capillaries by cellular outgrowth from existing microvessels (1) and occurs as part of the natural healing process following ischemic injury. Angiogenesis is associated with postinfarct remodeling and has important implications for prognosis following myocardial infarction. Therefore, the noninvasive evaluation of angiogenesis may help predict left ventricular remodeling and permit risk stratification of patients following myocardial infarction. In addition, newer therapies for treatment of ischemic heart disease involve stimulation of this natural response. Many approaches to therapeutic myocardial angiogenesis have been explored, including gene therapy, intramyocardial administration of angiogenic factors, and transmyocardial revascularization (2-10). While treatment with factors such as VEGF and bFGF has produced encouraging results in animal models (2-4) and in early clinical trials $(7,11)$, double-blind, placebo-controlled clinical studies to date have resulted in less-favorable outcomes $(8,10)$. These seemingly contradictory findings may be directly related to the limitations of existing clinical methods directed at noninvasive evaluation of

Nonstandard abbreviations used: indium-111 ( $\left.{ }^{111} \mathrm{In}\right)$; iodine-125 ( $\left.{ }^{125} \mathrm{I}\right)$; left anterior descending (LAD); magnetic resonance (MR); 2-methoxy-2-methylpropyl-isonitrile (sestamibi); positron emission tomography (PET); single-photon emission-computed tomographic (SPECT); Technetium-99m $\left({ }^{99 \mathrm{~m} T c)}\right.$; thallium-201 $\left({ }^{201} \mathrm{Tl}\right) ; 2,3,5$-triphenyl2H-tetrazolium chloride (TTC).

Conflict of interest: D.S. Edwards, S. Liu, and T.D. Harris were employees of BristolMyers Squibb at the time the studies were performed.

Citation for this article: J. Clin. Invest. 113:1684-1691 (2004).

doi:10.1172/JCI200420352. the physiological effects of angiogenesis, which may be very focal or nontransmural and therefore difficult to detect noninvasively. Unfortunately, standard cardiac noninvasive imaging strategies have not been effective in evaluation of cardiac angiogenesis (12). The development of more sensitive and specific imaging strategies for the direct evaluation of myocardial angiogenesis, and tracking this process noninvasively, will be critical in evaluation of patients following myocardial infarction and in assessing new angiogenic therapy for the heart.

The angiogenic response is modulated by the composition of the ECM and intercellular adhesions, including integrins (13, 14). Integrins are a family of heterodimeric cell surface receptors capable of mediating an array of cellular processes, including cell adhesion, migration, proliferation, differentiation, and survival (15). During angiogenesis endothelial cells must adhere to one another and to the ECM in order to construct and extend new microvessels. The specific $\alpha v \beta 3$ integrin has been identified as a critical modulator of angiogenesis (14).

Angiogenic vessels demonstrate increased expression of the $\alpha v \beta 3$ integrin; therefore the $\alpha v \beta 3$ integrin represents a potential novel target for directly imaging angiogenesis $(16,17)$. Investigators have proposed the potential noninvasive detection of tumor angiogenesis in vivo using magnetic resonance imaging and a paramagnetic contrast agent targeted to endothelial $\alpha v \beta 3$ via the $\mathrm{mAb}$ for the $\alpha v \beta 3$ integrin (LM609) (16). Haubner et al. reported the synthesis and favorable imaging characteristics of a iodine-125-labeled ( ${ }^{125}$ I-labeled) $\alpha v \beta 3$ antagonist (17-19). Harris et al. recently reported the high affinity and selectivity of an indium-111-labeled quinolone ( ${ }^{111}$ In-RP748) for the $\alpha v \beta 3$ 

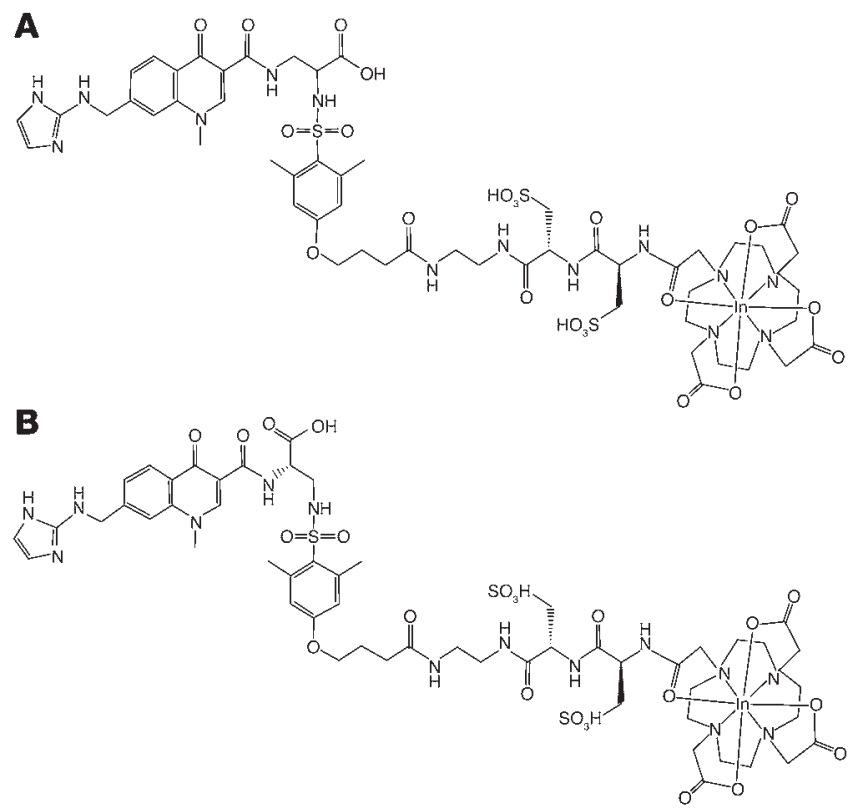

Figure 1

Structure of ${ }^{111} \mathrm{In}-\mathrm{RP} 748$, a quinolone targeted at $\alpha \mathrm{v} \beta 3$ integrin (A), and control compound (B).

integrin using assays of integrin-mediated adhesion (20). These investigators also demonstrated a rapid blood clearance and favorable biodistribution of ${ }^{111}$ In-RP748 and the feasibility for tumor imaging. We recently demonstrated that ${ }^{111} \mathrm{In}-\mathrm{RP} 748$ binds preferentially to activated $\alpha v \beta 3$ on endothelial cells in vitro and exhibits favorable binding characteristics for in vivo imaging (21). These preliminary in vitro studies and work in imaging tumor angiogenesis supports the potential for radiolabeled targeting of $\alpha v \beta 3$ for imaging of myocardial angiogenesis.

We hypothesized that the angiogenic process in the heart can be directly tracked noninvasively by single-photon emission-computed tomographic (SPECT) imaging of radiolabeled ligands targeted at the $\alpha v \beta 3$ integrin. The purpose of the current study was to evaluate ${ }^{111}$ In-RP748, a radiolabeled quinolone targeted at $\alpha v \beta 3$, for imaging of ischemia-induced angiogenesis following ischemic injury in established rodent and canine models of myocardial infarction. The chemical structure of ${ }^{111}$ In-RP748 is shown in Figure 1A. We evaluate the uptake of ${ }^{111}$ In-RP748 following ischemic injury, in relation to immunohistochemical markers of the angiogenic process and myocardial perfusion. The specificity of myocardial ${ }^{111}$ In-RP748 uptake was demonstrated in a chronic rodent model of injury-induced myocardial angiogenesis using paired control experiments with an analogue compound (Figure 1B), which demonstrated no in vitro specificity for the $\alpha v \beta 3$ integrin. Subsequent studies employing a chronic canine model of injury-induced myocardial angiogenesis demonstrated the feasibility of SPECT imaging for noninvasive detection of the $\alpha v \beta 3$ integrin.

\section{Methods}

All experimental studies were performed with approval of the Institutional Animal Care and Use Committee, according to the guiding principles of the American Physiological Society on research animal use.

\section{Rodent experiments}

Rodent surgical preparation. A chronic rat model of infarction was employed using procedures modified from those previously reported (22). Male Sprague-Dawley rats (200-250 g) were anesthetized with isoflurane, and the hearts were exposed by a limited left anterolateral thoracotomy. A 6-0 proline ligature was used to occlude the left anterior descending (LAD) coronary artery $7 \mathrm{~mm}$ distal to its origin. Serial brief preconditioning occlusions were performed to suppress ventricular dysrhythmias, followed by a 45 minute experimental occlusion. The coronary occlusion was then released, and the chest was closed in layers.

Rodent experimental protocol. Two weeks after myocardial infarction, rats $(n=11)$ were injected intravenously with either ${ }^{111} \mathrm{In}$ RP748 $(1.2 \pm 0.1 \mathrm{mCi})$, an agent targeted at the $\alpha v \beta 3$ integrin, or ${ }^{111}$ In-RP790 (1.2 $\left.\pm 0.1 \mathrm{mCi}\right)$, a nonspecific control agent. Ninety minutes after injection of one of the ${ }^{111} \mathrm{In}$-labeled agents, rats were injected with thallium-201 $\left({ }^{201} \mathrm{Tl} ; 0.78 \pm 0.06 \mathrm{mCi}\right)$ for evaluation of relative myocardial perfusion. Rats were euthanized 30 minutes after ${ }^{201} \mathrm{Tl}$ injection for postmortem analysis of myocardial tissue activity. The myocardial uptake of the ${ }^{111}$ In-labeled agents was compared to relative ${ }^{201} \mathrm{Tl}$ perfusion and immunohistochemical markers of the angiogenic process.

Postmortem analysis of rat hearts. Rodent hearts were excised and filled with dental molding material (alginate impression material, type II - normal set; Quala Dental Products, Milford, Delaware, USA) to facilitate cutting of the hearts into uniform 2$\mathrm{mm}$-thick slices. Selected slices were frozen and sectioned for immunohistochemistry of both lectin (marker of endothelium) $\alpha \mathrm{v}$ and $\beta 3$. Unstained heart slices were then cut into eight transmural segments for $\gamma$-well counting of ${ }^{201} \mathrm{Tl}$ and ${ }^{111} \mathrm{In}$ activity, using two energy windows ( ${ }^{201} \mathrm{Tl}$ : $60-90 \mathrm{keV} ;{ }^{111} \mathrm{In}: 170-300 \mathrm{keV}$ ). Raw counts were corrected for spill-up/spill-down, background, decay, and weight. The corrected counts were normalized to a nonischemic region of the heart, and the segments were segregated by myocardial ${ }^{201} \mathrm{Tl}$ retention into one of four groups: $0-40 \%, 41-60 \%, 61-80 \%$, or $80-120 \%$ nonischemic.

\section{Canine experiments}

Studies were also performed in dogs $(n=6)$ with injury-induced myocardial angiogenesis in order to better define the expression of the $\alpha v \beta 3$ integrin in the region of myocardial injury, to better localize the uptake of ${ }^{111}$ In-RP748 in relationship to myocardial injury and perfusion, and to establish the feasibility of in vivo imaging of myocardial angiogenesis with ${ }^{111}$ In-RP748.

Canine surgical preparation. Six fasted adult mongrel dogs were injected with $10 \mathrm{mg} / \mathrm{kg}$ thiopental sodium intravenously and were intubated. Animals were placed on a respirator and mechanically ventilated with nitrous oxide and oxygen (3:1) and $0.5-1.5 \%$ halothane. Dogs underwent cardiac catheterization via the right femoral artery, using a standard coronary guide catheter, and a baseline angiogram was obtained. Either the proximal LAD or left circumflex coronary artery was occluded for 2 hours using a balloon angioplasty catheter (diameter: $2.5 \mathrm{~mm}$; length: $20 \mathrm{~mm}$ ) under fluoroscopic guidance. The balloon was inflated to a pressure of $4 \mathrm{~atm}$. An angiogram was performed immediately after inflation and every 15 minutes thereafter to confirm coronary occlusion. The coronary occlusion was released after 2 hours, and coronary reflow was confirmed angiographically. An angiogram was performed prior to each nuclear-imaging session to confirm vessel patency. 


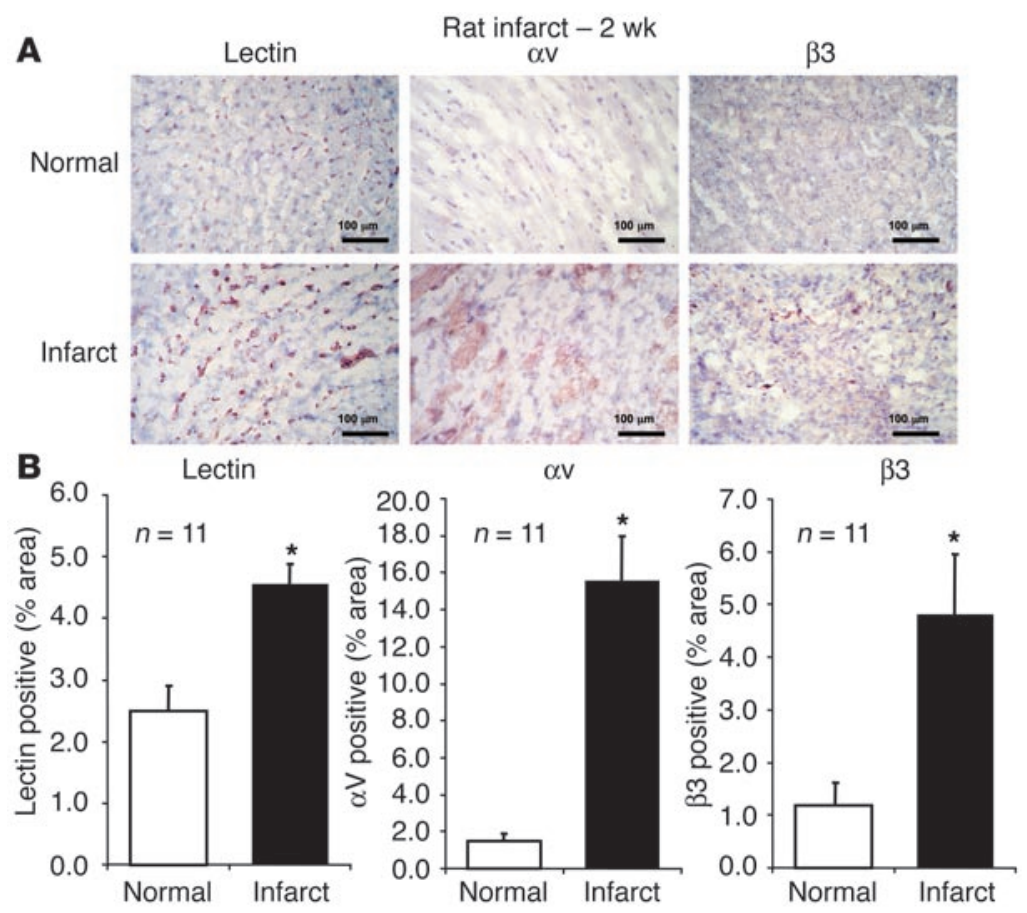

Figure 2

Immunohistochemical analysis in chronic rat model. Example of lectin, $\alpha v$, and $\beta 3$ staining is shown for normal and infarct region $(\mathbf{A})$ in rats 2 weeks following infarction. The infarct region demonstrated an increase in capillary density and arterioles, as well as increased staining for $\alpha v$ and $\beta 3$. This was confirmed by quantitative analysis (B). ${ }^{*} P<0.05$ vs. normal.

In vivo SPECT imaging of ${ }^{111}$ In-labeled $\alpha v \beta 3$ and perfusion. All dogs $(n=6)$ underwent dual isotope SPECT imaging with ${ }^{111}$ In-RP748 and Technetium-99m-labeled (99mTc-labeled) 2-methoxy-2-metholpropyl-isonitrile (sestamibi). Dogs were imaged following 6-10 hours of reperfusion, as well as at 1 and 3 weeks after reperfusion. Following intravenous injection of ${ }^{111} \mathrm{In}-\mathrm{RP} 748(6.6 \pm 1.0 \mathrm{mCi})$, six serial 15 -minute SPECT images were acquired starting at 15 minutes after injection. The last SPECT acquisition was initiated 90 minutes after injection. Images were acquired using two energy windows, $180 \mathrm{keV} \pm 7.5 \%$ and $252 \mathrm{keV} \pm 10 \%$, grouped into a single image. All nuclear imaging was done on a Dual-Head Gamma Camera (GE Millennium; General Electric Corp., Waukesha, Wisconsin, USA), using medium energy parallel-hole collimators. SPECT images were acquired in continuous advance mode, 15 seconds per $3^{\circ}$ frame, with a zoom of 1.77 and $64 \times 64$ matrix. Following the ${ }^{111} \mathrm{In}$-RP748 SPECT imaging, $22.3 \pm 5.4 \mathrm{mCi}$ of ${ }^{99 \mathrm{~m}}$ Tc-sestamibi was injected. A 15 -minute SPECT $99 \mathrm{~m}$ Tc-sestamibi image was acquired 15 minutes after injection to provide a reference perfusion image facilitating reconstruction of the ${ }^{111}$ In-RP748 "hot spot" image. Acquisition parameters were identical to those used for the ${ }^{111}$ In-RP748 SPECT acquisitions, except for an energy window center at $140 \mathrm{keV}( \pm 7.5 \%)$.

All SPECT images were batch reconstructed using standard filtered back projection (order 4 , cutoff $0.28 \times$ Nyquest frequency) without attenuation or scatter correction. To quantify the relative signal-to-noise ratio in our reconstructed SPECT images, equal-size regions of interest were drawn over the targeted hot spot and remote myocardial regions. The remote myocardial region was localized within the myocardium on the targeted image based on a location defined by the registered perfusion images.

Postmortem analysis in canine model. Three weeks after reperfusion, following the last imaging session, dogs were euthanized and hearts rapidly excised. Transmural myocardial sections were cut from the normal and central infarct regions of the heart, and tissue from each biopsy was split into two transmural sections. One half was fixed in $10 \%$ formalin solution and later embedded in paraffin. The other half of each biopsy was frozen in embedding compound (Tissue-Tek OCT; Sakura Finetek USA Inc., Torrance, California, USA) using a bath of 2-methylbutane cooled on dry ice.

Ex vivo imaging of ${ }^{111}$ In-labeled $\alpha v \beta 3$ and perfusion. After left ventricle tissue was obtained from biopsies, the heart was cast with dental impression material and sliced into uniform short axis slices $(5 \mathrm{~mm})$. Cast slices were placed in direct contact with the medium energy collimator surface of the gamma camera in order to obtain registered sequential high-resolution $(256 \times 256){ }^{99 \mathrm{~m} T c-s e s t a m i b i}$ and ${ }^{111} \mathrm{In}$-RP748 images using the previously defined energy windows.

Postmortem analysis of canine hearts. Heart slices were stained with a buffered solution of 2,3,5-triphenyl-2Htetrazolium chloride (TTC) at $38^{\circ} \mathrm{C}$ to identify myocardial infarction. Digital photographs were taken of both the apical and basal surfaces of all stained slices.

Heart slices were stripped of epicardial fat and surface vessels, cut into eight radial pies, and each pie was divided transmurally into epicardial and endocardial pieces for $\gamma$-well counting using two energy windows $\left({ }^{99 \mathrm{~m}} \mathrm{Tc}\right.$ : 130-150 keV; ${ }^{111}$ In: $\left.170-300 \mathrm{keV}\right)$. Raw $\gamma$ counts were corrected for background, decay, spill-up/spill-down and were expressed as counts per minute per gram of tissue. Activity in each myocardial sample was normalized to the activity in a nonischemic region of the heart. Tissue activities were grouped according to normalized ${ }^{99 \mathrm{~m} T c}$-sestamibi activity into one of four groups: $0-40 \%, 41-60 \%$, $61-80 \%$, or $81-120 \%$ nonischemic.

\section{Histological and immunohistochemical analysis}

Formalin-fixed myocardial sections were stained with Masson's trichrome using standard techniques to define the area of fibrosis. Cryostat sections ( $5 \mu \mathrm{m}$ thick) were used for immunohistochemical staining. For our immunohistochemical analysis of rat myocardial angiogenesis, sections stained with a biotinylated endothelial-specific lectin Bandeiraea Simplicifolia Lectin I (Vector Laboratories, Burlingame, California, USA). Since no specific $A b$ is available for rat $\alpha v \beta 3$ integrin, we performed separate staining for $\alpha v$ (rabbit anti-integrin $\alpha v$ subunit polyclonal $\mathrm{Ab}$; Chemicon International, Temecula, California, USA) and $\beta 3$ (mouse anti-rat integrin $\beta 3 \mathrm{mAb}$; BD Biosciences PharMingen, San Diego, California, USA) integrins. Sections were then incubated with respective biotinylated secondary Ab's. The signal was detected using HRP avidin (Elite ABC VectaStain Kit; Vector Laboratories). The peroxidase label was developed using 3-(2-aminoethyl)carbazole (Red AEC Kit; Vector Laboratories), and the sections were counterstained with hematoxylin. Analysis of angiogenesis in dogs was accomplished with the 
A
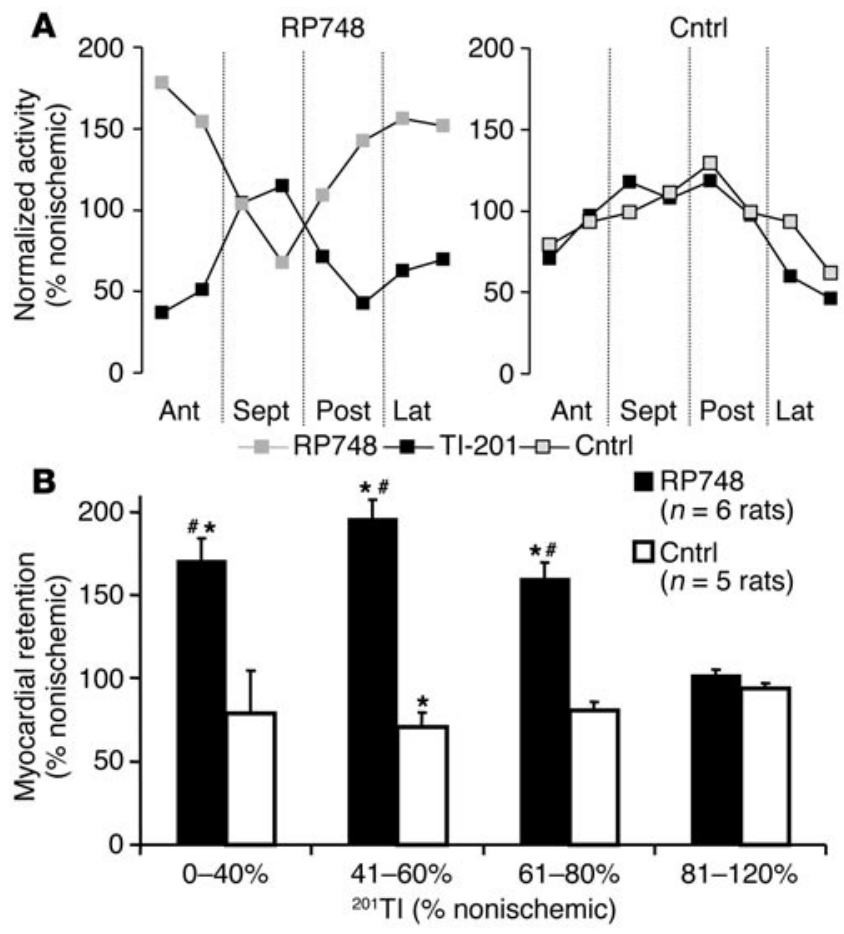

biotinylated endothelial-specific lectin, Bandeiraea Simplicifolia Lectin I (Vector Laboratories). For immunostaining of the $\alpha v \beta 3$ integrin in dogs, we used a specific mouse mAb for the $\alpha v \beta 3$ integrin (LM609; Chemicon International).

Sections were analyzed quantitatively for extent (percentage of area) of positive staining using computer algorithms developed and validated in our laboratory, which take into account the image hue, saturation, and intensity color map. Quantitative values for infarct and control regions in each animal were calculated by averaging results for four representative high-powered fields $(\times 200)$ in each region.

\section{Canine cell culture studies}

Canine femoral vein endothelial cells were isolated by scraping and were cultured as previously described (21). A cy3-labeled homologue (TA145) of ${ }^{111}$ In-RP748 was used to confirm and localize endothelial cell binding. The localization of TA145 was compared with that of LM609. LM609 is a mAb for the $\alpha v \beta 3$ integrin, which has been previously demonstrated to bind to canine microvascular endothelial cells on frozen tissue sections from reperfused infarcted regions (23). The localization of TA145 and LM609 was also compared with an isotype-matched control Ab of LM609 (MOPC21).

Figure 4

Immunohistochemical analysis in chronic canine model. Masson's trichrome staining demonstrated increased vascular density in the fibrotic central infarct region, which was confirmed by lectin staining. The infarct region demonstrated increased staining for $\alpha v \beta 3$ using the LM609 Ab (A). LM609 staining localized to the endothelial cells of capillaries and endothelial cells, as well as smooth muscle cells of small arterioles within the infarct region. Very little LM609 staining was in seen in remote noninfarcted regions. These regional differences in staining were confirmed by quantitative analysis (B). ${ }^{*} P<0.05$ vs. normal.

\section{Figure 3}

$\gamma$-Well counting of myocardial radiotracer activity in relationship to ${ }^{201} \mathrm{TI}$ uptake in the chronic rat model. Data are shown for rats injected with either ${ }^{111}$ In-RP748 (RP748) or control compound. Decreased myocardial ${ }^{201} \mathrm{TI}$ uptake was consistently observed in the anterolateral wall, as shown in representative myocardial count profiles (A). Cntrl, control; ant, anterior; sept, septal; post, posterior; lat, lateral. Uptake of ${ }^{111}$ In-RP748 was highest in infarcted regions with reduced ${ }^{201} \mathrm{TI}$ retention. In contrast, myocardial uptake of the control compound tracked ${ }^{201} \mathrm{TI}$ perfusion. On average the relative myocardial retention of ${ }^{111} \mathrm{In}-\mathrm{RP} 748$ in the postischemic and infarcted regions was nearly twice that in regions with normal ${ }^{201} \mathrm{TI}$ perfusion; however, no selective retention of the nonspecific control compound was observed (B). ${ }^{*} P<0.05$ vs. $81-120 \%$; $\# P<0.05$ vs. control.

\section{Statistical analysis}

All data are presented as mean plus or minus SD. Comparisons between two groups were made using either a paired or unpaired Student's $t$ test. Differences between groups were considered significant at $P$ values less than 0.05 (two-tailed).

\section{Results}

\section{Rodent chronic myocardial infarct experiments}

Studies were performed in rats $(n=11)$ with injury-induced myocardial angiogenesis. Immunohistochemical staining with an endothelial-specific lectin confirmed angiogenesis in the infarct region. In the absence of a specific anti-rat $\alpha v \beta 3 \mathrm{Ab}, \alpha \mathrm{v}$ and $\beta 3$

\section{A}
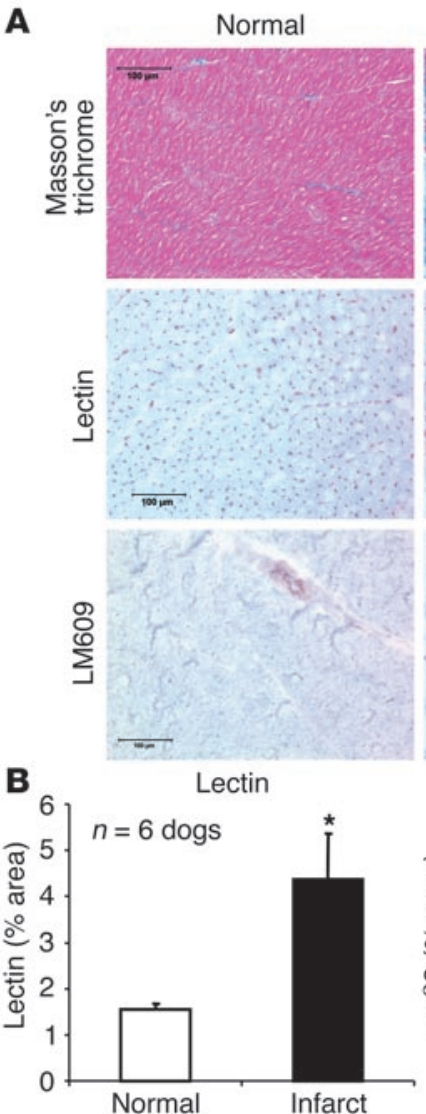

Infarct
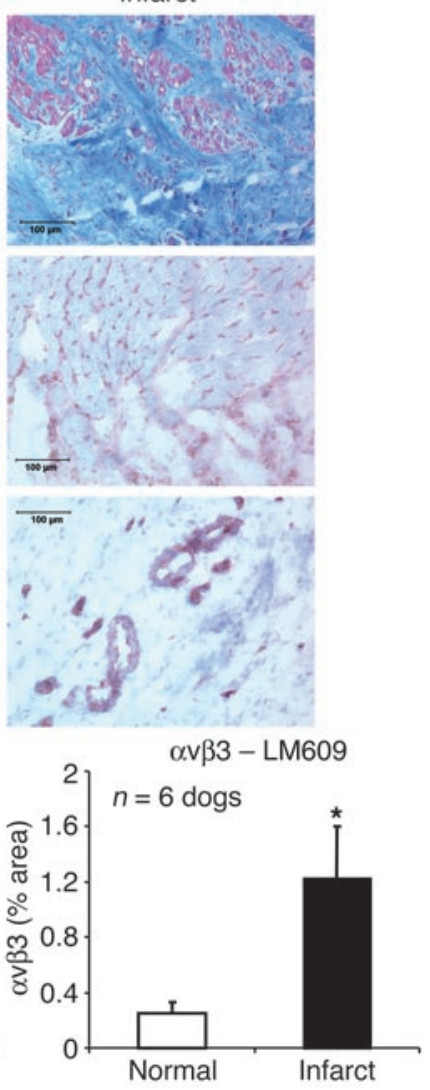
A

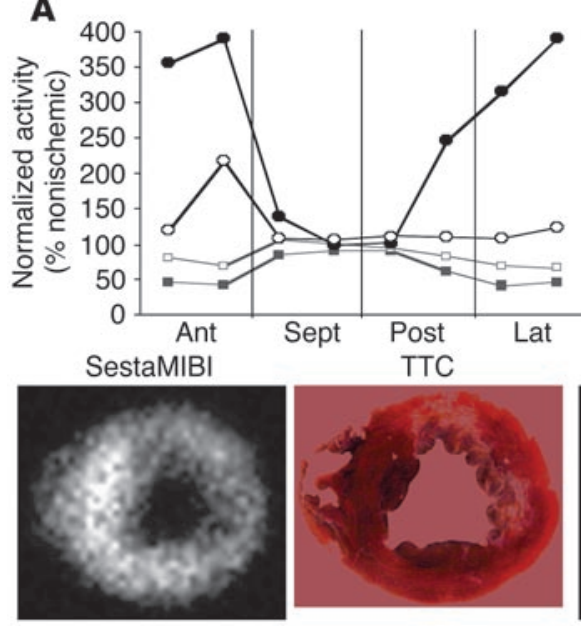

B

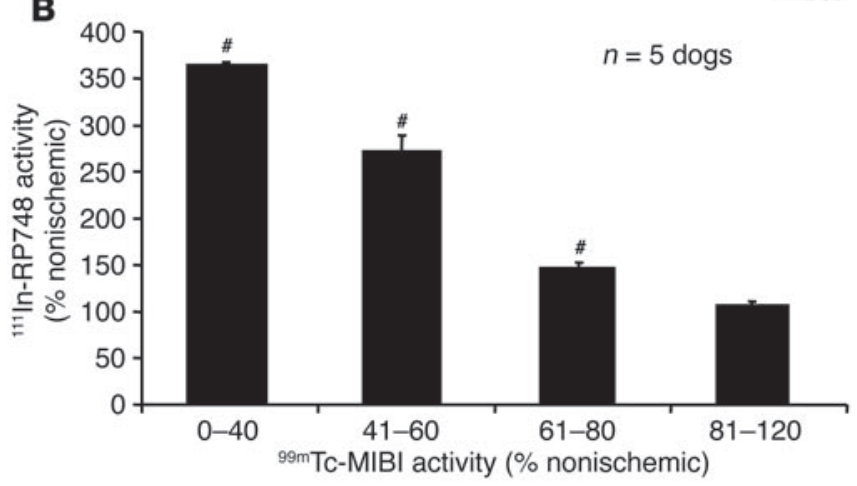

staining in this same region were performed and demonstrated increased expression of these integrins (Figure 2A). These changes were confirmed by semiautomated quantitative analysis of the immunohistochemical stains (Figure 2B). Following in vivo injection of ${ }^{111} \mathrm{In}-\mathrm{RP} 748$ and the perfusion tracer thallium-201 $\left({ }^{201} \mathrm{Tl}\right)$, $\gamma$-well counting of myocardial segments allowed quantification of relative myocardial ${ }^{111}$ In-RP748 retention in relationship to myocardial perfusion. The ${ }^{111} \mathrm{In}-\mathrm{RP} 748$ was selectively retained in the infarcted regions with reduced ${ }^{201} \mathrm{Tl}$ perfusion (Figure 3A). A twofold increase in the retention of ${ }^{111}$ In-RP748 was observed in most ischemic regions at 2 weeks after infarction (Figure 3B). In contrast, a nonspecific control compound demonstrated no preferential retention in the infarct region. The specific retention of ${ }^{111}$ In-RP748 in myocardial regions with injury-induced angiogenesis suggests that ${ }^{111}$ In-RP748 might be a candidate radiotracer for the noninvasive imaging of myocardial angiogenesis.

\section{Canine chronic myocardial infarct experiments}

Immunohistochemical staining of endotbelium and $\alpha \nu \beta 3$ integrin. Representative immunohistochemical stains are shown in Figure 4A. Lectin histochemical staining for endothelium confirmed angiogenesis in the central infarct region of the chronically infarcted dogs. Immunohistochemical staining with LM609, a specific Ab for the $\alpha v \beta 3$ integrin, also confirmed increased expression of the receptor in capillaries and of both the endothelium and smooth musculature of arterioles within the infarct region. Increased capillary staining was primarily seen in the peri-infarct region, while arteriolar smooth muscle staining with LM609 was observed within the central infarct

\section{Figure 5}

Well counting of myocardial radiotracer activity in relationship to 99mTc-sestamibi perfusion in chronic canine infarct model. Ex vivo 99mTc-sestamibi perfusion (left) and ${ }^{111} \mathrm{In}-\mathrm{RP} 748$ (right) images of representative myocardial slices from an infarcted dog, 3 wks after reperfusion, with the corresponding TTC-stained section (middle) (A). Corresponding circumferential ${ }^{99 m} \mathrm{Tc}$-sestamibi (MIBI) and ${ }^{111} \mathrm{In}-\mathrm{RP} 748$ count profiles are shown for both endocardial (ENDO) and epicardial (EPI) segments. Focal uptake of ${ }^{111} \mathrm{In}$-RP748 is seen in infarct region on ex vivo images, and is confirmed by $\gamma$-well counting. Myocardial segments from all dogs were segregated into four categories based on relative $99 \mathrm{~m} T \mathrm{~T}$-sestamibi perfusion (percentage nonischemic). Regional myocardial ${ }^{111}$ In-RP748 activity (percentage nonischemic) was significantly increased 3 wks after reperfusion in the ischemic regions of all dogs (B). ${ }^{\#} P<0.05$ vs. 81-120.

region. Quantitative analysis demonstrated a significant increase in vascular staining in the infarct region and increased activation of the $\alpha v \beta 3$ integrin in angiogenic vessels (Figure 4B). The relative increase in LM609 staining in the ischemic region (ischemic/nonischemic ratio) observed in the dogs significantly correlated $(r=0.84, P \leq 0.05)$ with the relative increase in myocardial uptake of ${ }^{111}$ In-RP748 within these same ischemic regions.

Postmortem tissue analysis in canine model. $\gamma$-Well counting of canine myocardial segments allowed quantification of the observed increase in relative myocardial ${ }^{111} \mathrm{In}-\mathrm{RP} 748$ retention in relationship to myocardial perfusion. Myocardial segments with decreased perfusion, as determined by ${ }^{99 \mathrm{~m}} \mathrm{Tc}$-sestamibi retention, demonstrate increased retention of ${ }^{111}$ In-RP748. Figure 5 illustrates the circumferential changes in endocardial and epicardial ${ }^{111}$ In-RP748 activity in relationship to ${ }^{99 \mathrm{~m}} \mathrm{Tc}$-sestamibi activity and corresponding ex vivo images in one of the dogs euthanized 3 weeks following infarction. Tissue $\gamma$-well counting data are summarized in Figure 5B. Dogs with chronic infarction demonstrated almost a fourfold increase in relative myocardial ${ }^{111} \mathrm{In}$-RP748 activity in the most ischemic regions as defined by ${ }^{99 \mathrm{~m}} \mathrm{Tc}$-sestamibi.

Dual-isotope in vivo SPECT imaging of ${ }^{111} I n-R P 748$ and ${ }^{99 m} T c$-sestamibi perfusion. The heart rate and aortic pressure remained stable in all chronically infarcted dogs that underwent serial dual-isotope in vivo SPECT imaging. The average hemodynamic values for these dogs are summarized in Table 1. Analysis of the in vivo ${ }^{111}$ In-RP748 SPECT images required registration and batch reconstruction of ${ }^{111}$ In-RP748 image data with ${ }^{99 \mathrm{~m}} \mathrm{Tc}$-sestamibi perfusion images. All dogs demonstrated focal myocardial retention of ${ }^{111}$ In-RP748 on in vivo SPECT imaging by 60 minutes after injection, at a time when blood pool activity had cleared. The increase in myocardial ${ }^{111}$ In-RP748 activity correlated with a ${ }^{99 \mathrm{~m}} \mathrm{Tc}$-sestamibi perfusion defect. The ${ }^{111} \mathrm{In}$-RP748 image quality was generally excellent, with a favorable heart-tobackground activity ratio. The targeted (hot spot) myocardial activity-to-remote background activity ratio varied from 1:1 to 1.6:1. A representative series of ${ }^{111} \mathrm{In}-\mathrm{RP} 748$ and corresponding

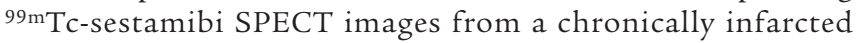
dog is shown in Figure 6. Serial dual-isotope ${ }^{111} \mathrm{In}-\mathrm{RP} 748$ and ${ }^{99 \mathrm{~m} T c-s e s t a m i b i}$ SPECT imaging demonstrated retention of ${ }^{111}$ In-RP748 in the infarct region (see Figure 6A). A representative dual-isotope ${ }^{111} \mathrm{In}$-RP748 and ${ }^{99 \mathrm{~m} T c-s e s t a m i b i}$ SPECT image series is shown in Figure 6B. Based on our analysis of the serial images, the maximum increase in myocardial ${ }^{111} \mathrm{In}-\mathrm{RP} 748$ retention appeared to occur at 1 week after infarction. 
A

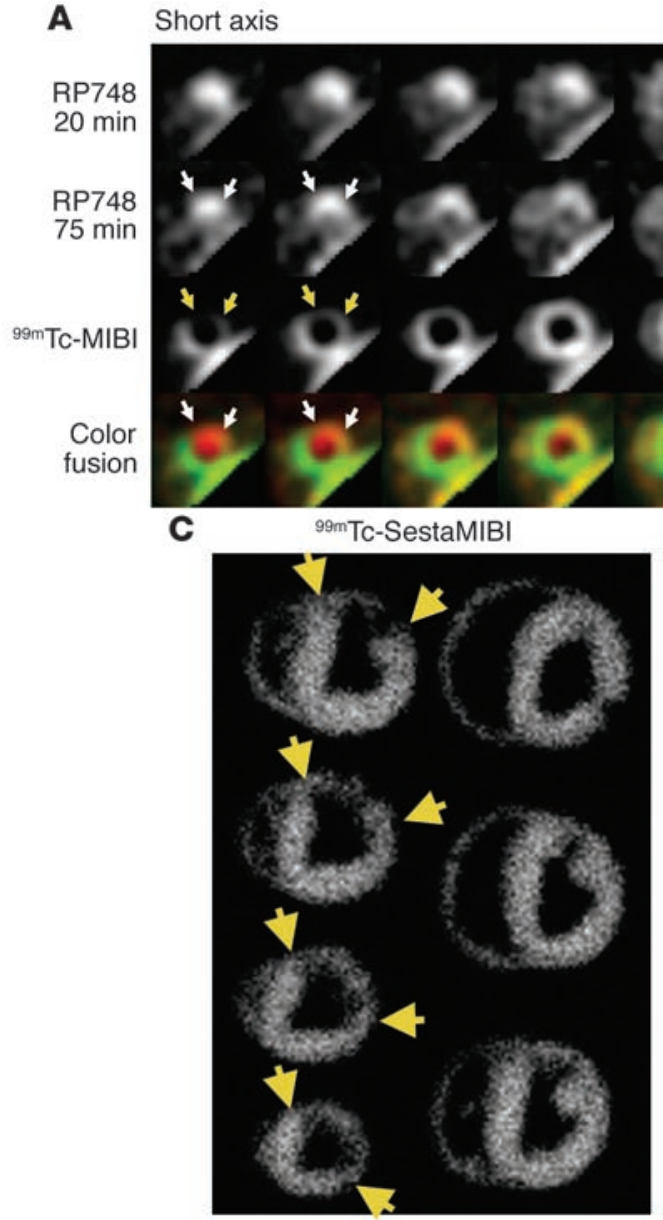

VLA

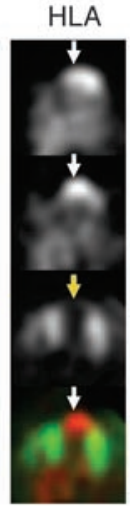

RP748

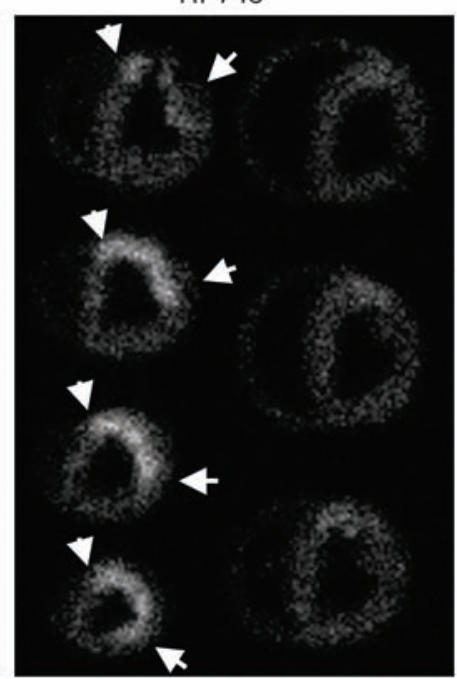

B
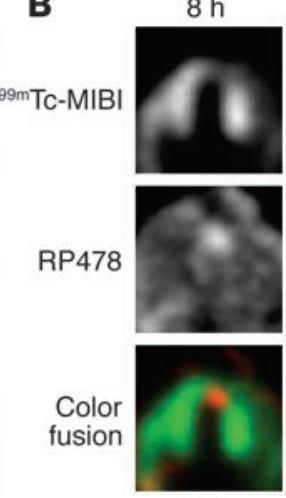

Fusion

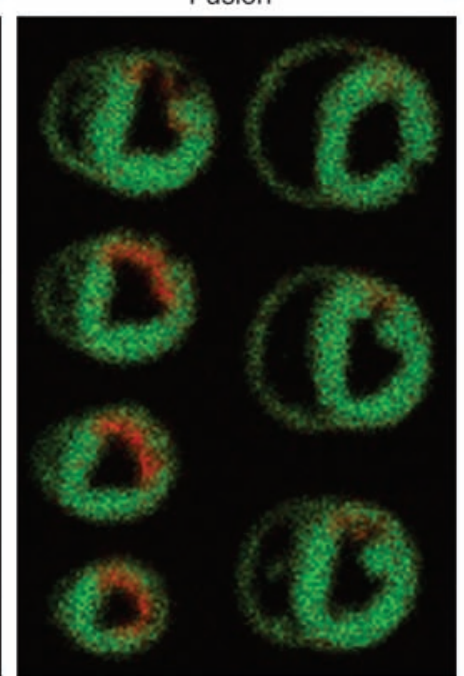

1 wk
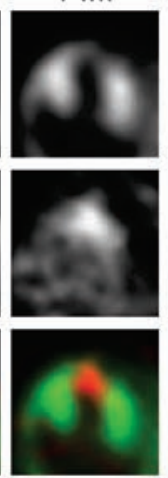

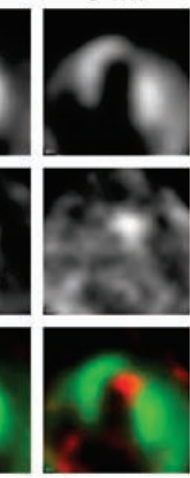

3 wk

\section{.}
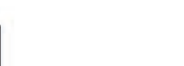

\section{Figure 6}

In vivo and ex vivo ${ }^{111} \mathrm{In}-\mathrm{RP} 748$ and ${ }^{99 \mathrm{mTC}}$-sestamibi (99mTc-MIBI) images from dogs with chronic infarction. Serial in vivo ${ }^{111} \mathrm{In}-\mathrm{RP} 748 \mathrm{SPECT}$ short axis, vertical long axis (VLA), and horizontal long axis (HLA) images in a dog 3 wks after LAD infarction at 20 min and 75 min after injec-

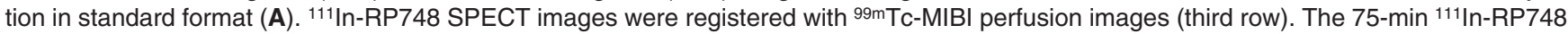

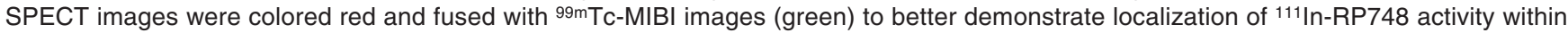
the heart (color fusion, bottom row). Right ventricular (RV) and left ventricular (LV) blood pool activity is seen at 20 min. White arrows indicate

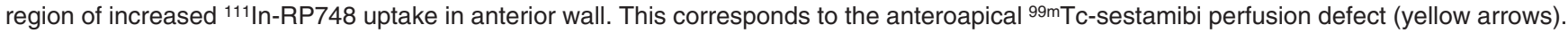
Sequential ${ }^{99 m T c}$-sestamibi (top row) and ${ }^{111} \mathrm{In}$-RP748 in vivo SPECT HLA images at 90 min after injection (middle row) from a dog at $8 \mathrm{~h}$ (acute), $1 \mathrm{wk}$, and 3 wks after LAD infarction (B). Increased myocardial ${ }^{111} \mathrm{In}-\mathrm{RP} 748$ uptake is seen in the anteroapical wall at all three time points. Color fusion ${ }^{99 \mathrm{~m} T c-M I B I}$ (green) and ${ }^{111} \mathrm{In}-\mathrm{RP} 748$ (red) images (bottom row) demonstrate ${ }^{111} \mathrm{In}-\mathrm{RP} 748$ uptake within ${ }^{99 \mathrm{mT}} \mathrm{Tc}-\mathrm{MIBI}$ perfusion defect. Ex

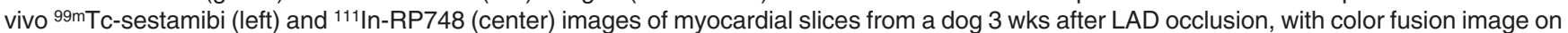
the right (C). Short axis slices are in the standard orientation. Yellow arrows indicate anterior location of nontransmural perfusion defect region; white arrows indicate corresponding area of increased ${ }^{111}$ In-RP748 uptake.

Dual-isotope ex vivo imaging of ${ }^{111}$ In-RP748 and ${ }^{99 m}$ Tc-sestamibi perfusion. The superior resolution of dual isotope ex vivo slice images allowed better localization of myocardial ${ }^{111}$ In-RP748 retention within the central infarct area. Figure $6 \mathrm{C}$ illustrates the focal uptake of ${ }^{111} \mathrm{In}-\mathrm{RP} 748$ within the nontransmural ${ }^{99 \mathrm{~m}} \mathrm{Tc}$-sestamibi perfusion defect. Myocardial retention of ${ }^{111}$ In-RP748 was apparent in the central infarct region. These images allowed direct correlation of myocardial ${ }^{111}$ In-RP748 retention with postmortem TTC staining for necrosis (see Figure 5A).

Canine cell culture studies. In cultured canine endothelial cells, a cy3-labeled analogue of ${ }^{111}$ In-RP748 (TA145) localized to $\alpha v \beta 3$ at focal cellular contacts in a distribution similar to LM609, an established $\mathrm{Ab}$ for $\alpha v \beta 3$ integrin (see Figure 7). This pattern of localization was not seen with an isotype-matched control Ab.
These in vitro data support that our radiolabeled compound does in fact bind to endothelial cells.

\section{Discussion}

The ${ }^{111}$ In-labeled $\alpha v \beta 3$-targeted agent ( ${ }^{111}$ In-RP748) may be a valuable targeted marker for noninvasive imaging of angiogenesis following nontransmural myocardial infarction. We employed established chronic rat and canine models of myocardial infarction, which are known to produce nontransmural infarction and peri-infarct ischemia resulting in myocardial angiogenesis. In our rat model of chronic infarction, ${ }^{111}$ In-RP748 was selectively retained in the infarcted regions with reduced ${ }^{201} \mathrm{Tl}$ perfusion. The specificity of ${ }^{111} \mathrm{In}$-RP748 retention in myocardial regions with ischemia-induced angiogenesis was supported by the use of 

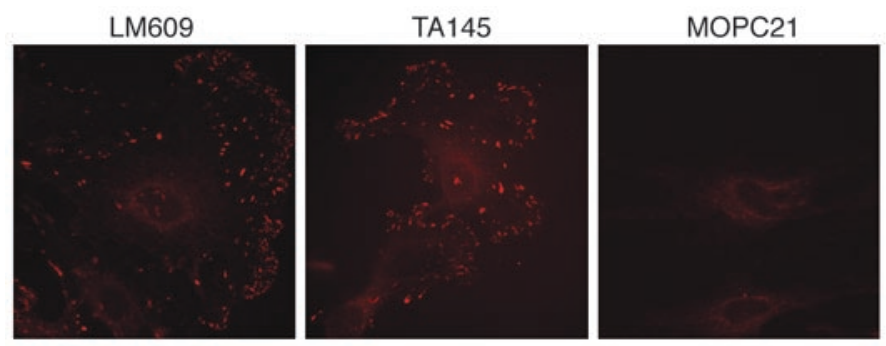

Figure 7

Evaluation of $\alpha v \beta 3$ integrin localization in cultured canine endothelial cells. LM609, an established $\mathrm{mAb}$ of $\alpha \mathrm{v} \beta 3$ integrin, is localized at cell contact points (left). A cy3-labeled analogue of ${ }^{111}$ In-RP748 (TA145) also localized to $\alpha v \beta 3$ at focal cellular contact points in a distribution similar to LM609 (middle). No focal cellular uptake was seen with the isotypematched negative control Ab (MOPC21, right).

a negative control compound, which demonstrated no increased uptake in the infarct region. These initial rat studies suggested that ${ }^{111}$ In-RP748 might be a candidate radiotracer for the noninvasive imaging of myocardial angiogenesis. The use of a canine model permitted the noninvasive serial evaluation of changes in myocardial ${ }^{11}$ In-RP748 uptake in relationship to changes of myocardial perfusion. The ${ }^{111}$ In-RP748 cleared rapidly from the blood and demonstrated a favorable biodistribution for imaging of myocardial angiogenesis. In vivo SPECT imaging demonstrated focal ${ }^{111}$ In-RP748 uptake in the infarct region associated with increased activation of the $\alpha v \beta 3$ integrin. Relative ${ }^{111} \mathrm{In}-\mathrm{RP} 748$ activity within the infarct was increased to $370 \%$ of nonischemic myocardium at 3 weeks after reperfusion. Analysis of serial in vivo SPECT images suggests that peak uptake of ${ }^{111} \mathrm{In}-\mathrm{RP7} 48$ may in fact occur closer to 1 week following reperfusion. Noninvasive imaging strategies similar to that employed in this study may be useful for evaluating angiogenesis and assessing efficacy of angiogenic therapies in patients with chronic ischemia or following myocardial infarction.

\section{Role of $\alpha \nu \beta 3$ integrin in myocardial angiogenesis}

Integrins are $\alpha \beta$ heterodimeric receptors that mediate divalent cation-dependent cell-cell and cell-matrix adhesion through tightly regulated interactions with ligands (24). The integrins are involved with many fundamental cellular processes, such as attachment, migration, proliferation, differentiation, and survival. The $\alpha v \beta 3$ integrin allows endothelial cells to interact with a wide variety of ECM components. The mAb against $\alpha v \beta 3$ (LM609) inhibits angiogenesis by selectively promoting apoptosis of vascular cells (13). Therefore, the $\alpha v \beta 3$ integrin plays a critical role in signaling events vital to survival of vascular cells undergoing angiogenesis. Enhanced expression of the $\alpha v \beta 3$ integrin has been previously observed in new blood vessels involved with angiogenesis (14). Endothelial cell migration is one of the critical steps in angiogenesis that specifically requires $\alpha v \beta 3$ integrins (25).

We observed increased expression of $\alpha v \beta 3$ integrin as late as 3 weeks following ischemic injury in endothelial and VSMCs within the central infarct area using immunohistochemical analysis with LM609. Brooks et al. suggested that peak expression of av $\beta 3$ occurs 12-24 hours after initiation of angiogenesis with bFGF (14). Sun et al. recently demonstrated increased expression of $\beta 3$ in rats following infarction. In these studies of permanent coronary occlusion, $\beta 3 \mathrm{mRNA}$ and protein peaked at 7 days after infarction and localized in the endothelial and smooth muscle cells in peri-infarct vessels (26). Additional experimental studies will be required to define the duration of $\alpha v \beta 3$ integrin expression and/or activation following ischemic injury and under conditions of stimulated angiogenesis.

\section{Imaging of $\alpha \nu \beta 3$ integrin}

Investigators have previously proposed noninvasive imaging of tumor angiogenesis with compounds specifically targeted at the $\alpha v \beta 3$ integrin, using magnetic resonance (MR) imaging (16), positron-emission tomography (PET) (19), and SPECT imaging (17). No prior studies have attempted $\alpha v \beta 3$ integrintargeted imaging of myocardial angiogenesis.

Targeted SPECT imaging of myocardial angiogenesis offers many advantages over clinical indices or imaging with other modalities. Rather than measuring an effect or consequence of angiogenesis, targeted SPECT imaging directly evaluates the angiogenic process. This is especially important considering the strong placebo effect that has been suggested in clinical trials of angiogenic factors. While clinical studies of angiogenic therapies have demonstrated minor increases in SPECT perfusion, we have demonstrated an approximate two- to fourfold increase in radiotracer uptake in regions of ischemia-induced myocardial angiogenesis. The dual-isotope SPECT perfusion and ${ }^{111}$ In-RP748imaging approach described in this study could easily be adapted for clinical imaging using existing equipment. In this study, we used an ${ }^{111}$ In-labeled quinolone targeted at $\alpha v \beta 3$ for imaging of ischemia-induced angiogenesis, which permitted serial in vivo SPECT imaging in a canine model of myocardial infarction. Image quality for clinical application could be further improved by use of a ${ }^{99 m}$ Tc-labeled compound targeted at the $\alpha v \beta 3$ integrin in combination with ${ }^{201} \mathrm{Tl}$ perfusion. This radiotracer combination would allow use of higher-resolution low-energy collimators. Absolute quantification of myocardial ${ }^{111}$ In-RP748 uptake will ultimately require correction for attenuation, scatter, and partial volume errors. These corrections will become feasible clinically with the availability of hybrid SPECT and x-ray computed tomography imaging systems that will allow for correction of these errors.

\section{Clinical implications}

Angiogenesis plays an important role in infarct healing and left ventricular remodeling following myocardial infarction, and infarct remodeling has important implications for the prognosis following myocardial infarction. Therefore, the noninvasive evaluation of angiogenesis may have important implications for prediction of left ventricular remodeling and risk stratification of patients following myocardial infarction. The ability to stimulate myocardial angiogenesis also has applications in the treatment of a host of cardiac disorders involving myocardial ischemia, includ-

\section{Table 1}

Hemodynamics, chronic canine studies

$\begin{array}{lrrr} & \text { Acute } & \text { Week 1 } & \text { Week 3 } \\ \text { Heart rate }(\mathrm{bpm}) & 102 \pm 19 & 98 \pm 19 & 92 \pm 24 \\ \text { Systolic AoP }(\mathrm{mmHg}) & 101 \pm 24 & 114 \pm 27 & 99 \pm 21 \\ \text { Diastolic AoP }(\mathrm{mmHg}) & 71 \pm 11 & 75 \pm 20 & 72 \pm 23\end{array}$

bpm, beats per minute; AoP, aortic pressure. 
ing chronic coronary artery disease and myocardial infarction. Numerous approaches to exogenously stimulate vessel growth in the myocardium have been explored $(2,5-10)$. While to date a great deal of effort has been focused on the development of strategies to stimulate angiogenesis in the heart, there remains the need for an effective, clinically feasible method for the evaluation of these therapeutic strategies. Most clinical studies have relied on improvement of clinical indicators (i.e., increased treadmill exercise times, reduction in anginal classification), or evaluation of the physiological consequences of angiogenesis, as indirect evidence of myocardial angiogenesis. SPECT, PET, or MR imaging is frequently used to detect changes in myocardial perfusion or function as an indirect physiological indicator of angiogenesis. While these imaging approaches are very useful in identifying regions of moderately to severely diminished perfusion, they may lack the sensitivity to discriminate the potentially small physiological changes that might be expected to result from stimulated angiogenesis in the myocardium. In animal models, immunohistochemical methods and vessel counting are the gold standard for measuring angiogenesis. Immunohistological staining of the endothelium is very effective in identifying blood vessels in a sample of myocardium. These methods involve harvesting of tissue samples and, of course, would be inappropriate for clinical use. Targeted noninvasive SPECT hot spot imaging of the angiogenic process, however, similar to that described here, may be more sensitive for assessing efficacy of angiogenic therapies in patients with chronic ischemia or following myocardial infarction and permit characterization of angiogenesis in humans.

Targeted SPECT hot spot imaging of myocardial angiogenesis with ${ }^{111}$ In-RP748 may provide increased sensitivity for noninvasive evaluation of angiogenesis over methods that evaluate the physiological consequences of angiogenesis. In experimental studies of angiogenesis, targeted SPECT imaging of the $\alpha v \beta 3$ integrin offers the ability to evaluate serially the angiogenic process over time in both small and large animal models. In our experimental models of ischemia-induced angiogenesis, we have also confirmed the increased expression of the $\alpha v \beta 3$ integrin with immunohistochemistry. While immunohistochemical techniques permit evaluation of angiogenesis, information on the time course of angiogenic processes can be obtained only by sacrificing many animals at various time points. Although we can not exclude the possibility of additional binding of ${ }^{111}$ In-RP748 to inflammatory cells within the infarct region, we have demonstrated clear specific binding of a cy3-labeled homologue of ${ }^{111}$ In-RP748 to focal cellular contact points in cultured endothelial cells.

In this study we have demonstrated the ability of ${ }^{111}$ InRP748 SPECT imaging for serial evaluation of injury-induced angiogenesis. Importantly, the imaging approaches developed for these models of chronic infarction may directly translate to evaluation of angiogenesis in patients.

\section{Acknowledgments}

This research was supported by a grant-in-aid from the American Heart Association (0050516N) and a grant from the NIH, National Heart, Lung, and Blood Institute (1R01 HL-65662), both to A.J. Sinusas. The radiotracer was provided by Bristol-Myers Squibb. We gratefully acknowledge the technical assistance of Xiao-Yu Hu, Ion Jovin, Jason Soares, Christopher Weyman, and Anjali Nath.

Received for publication October 21, 2003, and accepted in revised form April 20, 2004.

Address correspondence to: Albert J. Sinusas, Yale University School of Medicine, Nuclear Cardiology, 3FMP, PO Box 208017, New Haven, Connecticut 06520-8017, USA. Phone: (203) 7854915; Fax: (203) 737-1026; E-mail: Albert.Sinusas@yale.edu.
1. Battegay, E.J. 1995. Angiogenesis: mechanistic insights, neovascular diseases, and therapeutic prospects. J. Mol. Med. 73:333-346.

2. Giordano, F.J., et al. 1996. Intracoronary gene transfer of fibroblast growth factor-5 increases blood flow and contractile function in an ischemic region of the heart. Nat. Med. 2:534-539.

3. Lazarous, D.F., et al. 1995. Effects of chronic systemic administration of basic fibroblast growth factor on collateral development in the canine heart. Circulation. 91:145-153.

4. Unger, E., et al. 1994. Basic fibroblast growth factor enhances myocardial collateral flow in a canine model. Am. J. Physiol. 266:H1588-H1595.

5. Schofield, P.M., et al. 1999. Transmyocardial laser revascularisation in patients with refractory angina: a randomised controlled trial. [erratum 1999, 353:1714]. Lancet 353:519-524.

6. Allen, K.B., et al. 1999. Comparison of transmyocardial revascularization with medical therapy in patients with refractory angina. N. Engl. J. Med. 341:1029-1036.

7. Rosengart, T.K., et al. 1999. Angiogenesis gene therapy: phase I assessment of direct intramyocardial administration of an adenovirus vector expressing VEGF121 cDNA to individuals with clinically significant severe coronary artery disease. Circulation. 100:468-474.

8. Hendel, R.C., et al. 2000. Effect of intracoronary recombinant human vascular endothelial growth factor on myocardial perfusion: evidence for a dose-dependent effect. Circulation. 101:118-121.

9. Vale, P.R., et al. 2000. Left ventricular electromechanical mapping to assess efficacy of
phVEGF(165) gene transfer for therapeutic angiogenesis in chronic myocardial ischemia. Circulation. 102:965-974.

10. Udelson, J.E., et al. 2000. Therapeutic angiogenesis with recombinant fibroblast growth factor-2 improves stress and rest myocardial perfusion abnormalities in patients with severe symptomatic chronic coronary artery disease. Circulation. 102:1605-1610.

11. Losordo, D.W., et al. 1998. Gene therapy for myocardial angiogenesis: initial clinical results with direct myocardial injection of phVEGF165 as sole therapy for myocardial ischemia. Circulation. 98:2800-2804.

12. Simons, M., et al. 2000. Clinical trials in coronary angiogenesis: issues, problems, consensus: An expert panel summary. Circulation. 102:E73-E86. http:// circ.ahajournals.org/cgi/content/full/102/11/e73.

13. Brooks, P., et al. 1994. Integrin avb3 antagonists promote tumor regression by inducing apoptosis of angiogenic blood vessels. Cell. 79:1157-1164.

14. Brooks, P., Clark, R., and Cheresh, D. 1994. Requirements of vascular integrin avb3 for angiogenesis. Science. 264:569-571.

15. Schwartz, M.A., Schaller, M.D., and Ginsberg, M.H. 1995. Integrins: emerging paradigms of signal transduction. Annu. Rev. Cell Dev. Biol. 11:549-599.

16. Sipkins, D.A., et al. 1998. Detection of tumor angiogenesis in vivo by alphaVbeta3-targeted magnetic resonance imaging. Nat. Med. 4:623-626.

17. Haubner, R., et al. 1999. Radiolabeled alpha(v)beta3 integrin antagonists: a new class of tracers for tumor targeting. J. Nucl. Med. 40:1061-1071.

18. Haubner, R., et al. 2001. Glycosylated RGD-con- taining peptides: tracer for tumor targeting and angiogenesis imaging with improved biokinetics. J. Nucl. Med. 42:326-336.

19. Haubner, R., et al. 2001. Noninvasive imaging of alpha(v)beta3 integrin expression using 18Flabeled RGD-containing glycopeptide and positron emission tomography. Cancer Res. 61:1781-1785.

20. Harris, T.D., et al. 2003. Design, synthesis, and evaluation of radiolabeled integrin $v 3$ receptor antagonists for tumor imaging and radiotherapy. Cancer Biother. Radiopharm. 18:631-645.

21. Sadeghi, M., et al. 2004. Detection of injuryinduced vascular remodeling by targeting activated alpha $V$ beta 3 integrin in vivo. Circulation. In press.

22. McNulty, P., and Luba, M. 1995. Transient ischemia induces regional myocardial glycogen synthase activation and glycogen synthesis in vivo. Am. J. Physiol. 268:H364-H370.

23. Frangogiannis, N., et al. 2001. Induction and suppression of interferon-inducible (IP)-10 in reperfused myocardial infarcts may regulate angiogenesis. FASEB J. 15:1428-1430.

24. Xiong, J.P., et al. 2001. Crystal structure of the extracellular segment of integrin alpha Vbeta3. Science. 294:339-345.

25. Clyman, R. 1992. Beta 1 and beta 3 integrins have different roles in the adhesion and migration of vascular smooth muscle cells on extracellular matrix. Exp. Cell Res. 200:272-284.

26. Sun, M., et al. 2003. Temporal response and localization of integrins beta 1 and beta 3 in the heart after myocardial infarction: regulation by cytokines. Circulation. 107:1046-1052. 